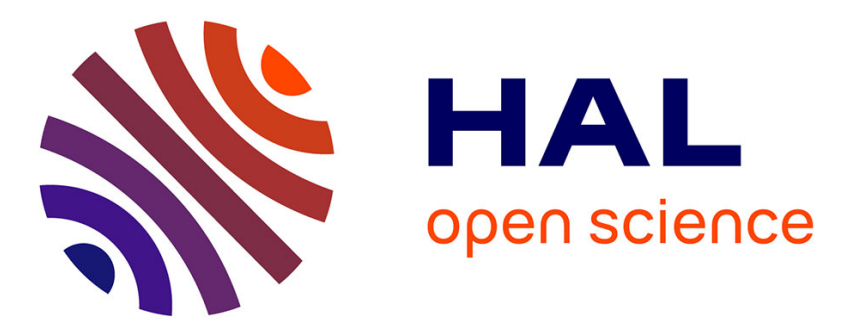

\title{
All-optical tunable pulse frequency chirp via slow light
}

\author{
G. Fanjoux, T. Sylvestre
}

\section{To cite this version:}

G. Fanjoux, T. Sylvestre. All-optical tunable pulse frequency chirp via slow light. Optics Letters, 2009, 34 (24), pp.3824-3826. 10.1364/OL.34.003824 . hal-00445301

\section{HAL Id: hal-00445301 \\ https://hal.science/hal-00445301}

Submitted on 5 May 2021

HAL is a multi-disciplinary open access archive for the deposit and dissemination of scientific research documents, whether they are published or not. The documents may come from teaching and research institutions in France or abroad, or from public or private research centers.
L'archive ouverte pluridisciplinaire HAL, est destinée au dépôt et à la diffusion de documents scientifiques de niveau recherche, publiés ou non, émanant des établissements d'enseignement et de recherche français ou étrangers, des laboratoires publics ou privés. 


\title{
All-optical tunable pulse frequency chirp via slow light
}

\author{
Gil Fanjoux* and Thibaut Sylvestre \\ Institut FEMTO-ST, Département d'Optique P. M. Duffieux, Université de Franche-Comté, \\ CNRS UMR 6174, Besançon, France \\ *Corresponding author: gil.fanjoux@univ-fcomte.fr
}

\begin{abstract}
We theoretically investigate slow light via stimulated Raman scattering, paying special attention to the picosecond regime where chromatic dispersion and cross-phase modulation must be considered. In addition to the control of the Raman pulse walk-off, we demonstrate that the cross-phase-modulation-induced frequency chirp can also be all-optically tuned via Raman slow light. We further demonstrate that this new implication is a consequence of the fact that the group velocity is significantly more affected than the phase velocity in slow-light media.
\end{abstract}

Slow and fast light (SFL) in nonlinear media has attracted considerable interest for several years and has been observed in plenty of configurations [1]. SFL generally requires a medium exhibiting sharp resonance with steep dispersion, which in turn results in a strong change of group velocity at the resonance, leading to optical delay or advancement. SFL can be achieved by using a medium with a frequencydependent absorption or gain or by using other mechanisms such as atomic resonances with narrow electromagnetically induced transparency (EIT) windows, coherent population oscillations (CPO), and laser-induced amplifying resonances [1]. For the development of fiber-based tunable delay lines at telecommunication wavelengths [2], SFL has also been studied in optical fibers with stimulated Brillouin scattering (SBS) [3,4] or stimulated Raman scattering (SRS) [5] or by using narrowband fiber parametric amplification [6].

The aim of this Letter is to stress that SFL affects light propagation in nonlinear material well beyond optical delay or advancement. SFL can indeed impact other physical parameters than group velocity, and, in particular, the pulse frequency chirp. In this Letter, we theoretically demonstrate in a slow-light Raman medium, where both group-velocity dispersion (GVD) and cross-phase modulation (XPM) must be considered, that the XPM-induced frequency shift can be all-optically controlled by tuning the pulse walk-off via slow light. Specifically, this is achieved by considering picosecond pulses propagating in a narrowband Raman fiber amplifier system that allows for the achievement of slow-light optical delays in the picosecond timescale. This could be implemented, for example, in a highly nonlinear hollowcore photonic crystal fiber [7] filled with carbon disulfide $\left(\mathrm{CS}_{2}\right)$, which is characterized by a strong and narrowband Raman gain enabling picosecond optical delays $[8,9]$.

Let us start our analysis by recalling the usual theory of the frequency chirp induced by a pump pulse on a small signal pulse at the Stokes Raman frequency through the combined effects of XPM and
GVD in an optical fiber. For normal GVD, the Stokes signal pulse predominantly interacts with the pump leading edge because of GVD-induced pulse walk-off, yielding an XPM-induced down-frequency shift. Assuming pump and signal Gaussian pulses synchronized at the medium input, the total frequency chirp across the signal pulse can be expressed at the waveguide output and in the signal reference frame as $[10,11]$

$$
\delta \nu(\tau)=-\frac{\gamma P L_{w}}{\pi T_{0}}\left[\exp \left(-\tau^{2}\right)-\exp \left(-\left(\tau-\delta_{w}\right)^{2}\right)\right]
$$

with $\gamma$ the nonlinear coefficient, $P$ the input pump power, $T_{0}$ the pulse width, $\tau=\left(t-z / v_{g s}\right) / T_{0}$ the normalized time, and $\delta_{w}=L / L_{w}$ with $L$ the fiber length. $L_{w}$ is the walk-off length expressed as $L_{w}=T_{0}\left(1 / v_{g p}\right.$ $\left.-1 / v_{g s}\right)^{-1}=L T_{0}\left(\Delta t_{L}\right)^{-1}$ with $v_{g p}$, and $v_{g s}$ the group velocity of the pump and the signal, respectively, with $\Delta t_{L}=L \Omega_{R} \beta_{2}$ the GVD temporal walk-off, where $\beta_{2}$ is the GVD coefficient and $\left(\Omega_{R} / 2 \pi\right)$ the Raman frequency detuning between the pump and the signal pulses [8]. The XPM-induced frequency shift at the signal pulse peak is then obtained by setting $\tau=0$ in Eq. (1),

$$
\delta \nu(0)=-\frac{\gamma P L_{w}}{\pi T_{0}}\left[1-\exp \left(-\delta_{w}^{2}\right)\right]
$$

Equation (2) yields a down-frequency shift linearly dependent on the pump power [10], as shown by the solid thin line in Fig. 1. This usual situation is also illustrated in the numerical simulation depicted in Fig. 2(a) that shows the normalized pump and signal pulses as well as the frequency chirp function, respectively. The inset of Fig. 2(a) shows the signal spectrum at the medium input and output, respectively, together with the associated down-frequency shift and the Raman gain band. The numerical simulation is based on the nonlinear Schrödinger equation (NLSE) including the Raman susceptibility, and the parameters are listed in the caption of Fig. 2. The real part of the Raman susceptibility has been delib- 


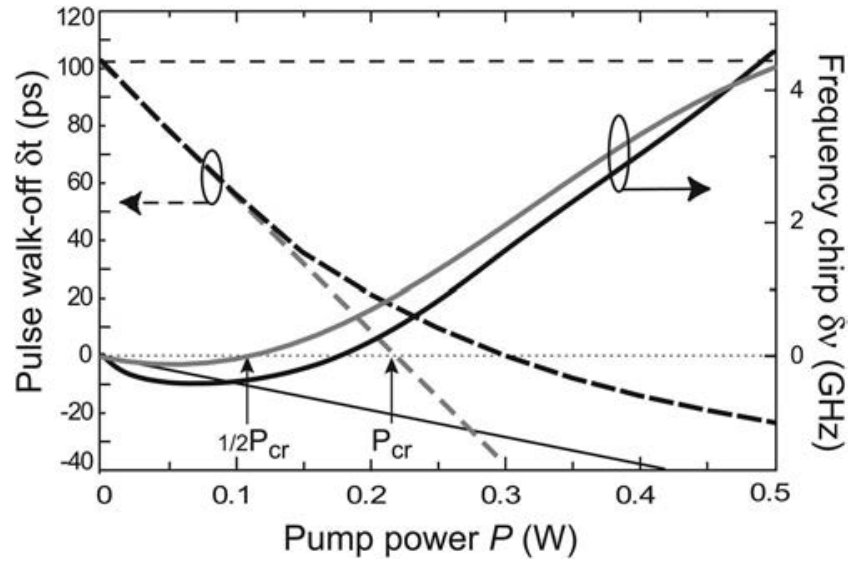

Fig. 1. Pulse walk-off (left, dashed) and XPM-induced frequency chirp (right, solid) as a function of the pump power $P$. Thin curve, standard theory without slow light, Eq. (2). Gray curve, analytical results with slow light [Eq. (4)]. Black curve, NLSE numerical results.

erately removed from the simulation so as not to include the slow-light effect [9].

If we now consider the slow-light process Eq. (2) is no longer valid, because slow light generates an additional tunable pulse walk-off and therefore leads to the full control of the signal pulse peak position with respect to both that of the pump pulse and of the XPM-induced chirp function. However the chirp function of Eq. (1) remains valid, because the slow light does not significantly affect the signal phase in our conditions, i.e., in a narrowband Raman amplifier in which the change of the group index is significantly larger than that of the phase index. It is indeed wellknown that the group velocity is much more affected by slow light than the phase velocity, since the former parameter is the derivative of the latter [1].

To take into account the new temporal position of the signal peak in the same reference frame as before (i.e., signal without slow light), we must define a normalized slow-light length as $L_{S L}=T_{0}\left(1 / v_{g s}^{S L}-1 / v_{g s}\right)^{-1}$ $=L T_{0}\left(\Delta t_{S L}\right)^{-1}$ where $v_{g s}^{S L}$ is the slow group velocity of the signal pulse. $\Delta t_{S L}=\left(g_{R} P L\right)\left(\Delta \Omega_{R} A_{\text {eff }}\right)^{-1}$ is the optical delay generated by Raman slow light, with $\left(\Delta \Omega_{R} / 2 \pi\right)$ the gain bandwidth, $g_{R}$ the Raman gain, and $A_{\text {eff }}$ the effective core area of the fiber [8]. Because of the slow-light optical delay, the temporal position of the signal envelope is now equal to $\tau$ $=L / L_{S L}=\delta_{S L}$ and not $\tau=0$, as in the previous case without slow light. The resulting XPM-induced frequency shift in the slow-light regime is thus given by

$$
\delta \nu\left(\delta_{S L}\right)=-\frac{\gamma P L_{w}}{\pi T_{0}} \times\left[\exp \left(-\delta_{S L}^{2}\right)-\exp \left(-\left(\delta_{S L}-\delta_{w}\right)^{2}\right)\right] .
$$

Compared to Eq. (2), Eq. (3) reveals that one can have full control of both the sign and the magnitude of the XPM-induced frequency chirp simply by tuning the pump power because of its new dependence on $\delta_{S L}$. This behavior is illustrated in Fig. 1, which shows the total pulse walk-off $\delta t=\Delta t_{L}-\Delta t_{S L}$ (dashed gray curve) and the associated XPM-induced fre-

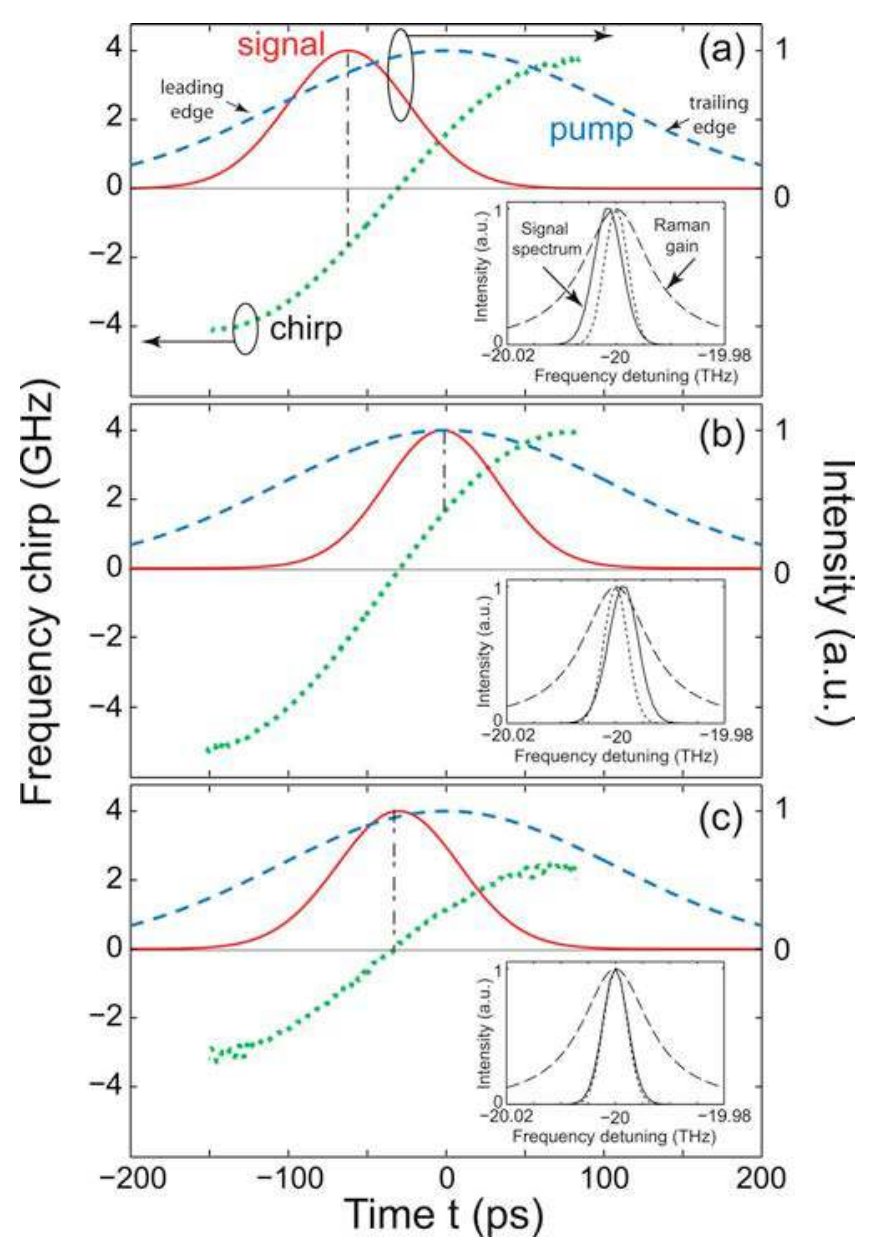

Fig. 2. (Color online) Frequency chirp $\delta \nu(\tau)$ (left, dotted curve) and pump and signal pulses profiles (dashed and solid normalized curves, respectively). The parameters are $T_{0}=250 \mathrm{ps}, \quad \gamma=4.134 \mathrm{~m}^{-1} . \mathrm{W}^{-1}, L_{w}=4.69 \mathrm{~m}, L=2 \mathrm{~m}$, $\left(\Omega_{R} / 2 \pi\right)=20 \mathrm{THz}, \quad\left(\Delta \Omega_{R} / 2 \pi\right)=15 \mathrm{GHz}, \quad A_{\text {eff }}=10 \mu \mathrm{m}^{2}, \quad \beta_{2}$ $=4.24 \times 10^{-25} \mathrm{~s}^{2} \mathrm{~m}^{-1}$, and $g_{R}=23.2 \times 10^{-11} \mathrm{~m} \mathrm{~W}^{-1}$ at the pump wavelength of $532 \mathrm{~nm}$. (a) Without slow light for $P$ $=P_{c r}$, similar to Eq. (1). (b) Zero walk-off for $P=P_{c r}$ $=0.285 \mathrm{~W}$. (c) Zero frequency shift for $P=0.17 \mathrm{~W}$. The insets show the signal spectrum at the medium's input (dotted curves) and output (solid curves), respectively, and the Raman gain (dashed curves).

quency chirp (solid gray curve), respectively, as a function of the pump power. Our analytical results have also been checked against numerical simulations (black curves in Fig. 1) that show the same tendencies for the walk-off and the frequency chirp, respectively. Figure 1 shows that the slow-light effect results in a pulse walk-off that decreases with the pump power, whereas in the absence it was constant, as recently demonstrated in [8]. More importantly, it also results on a frequency chirp that is predominantly positive, whereas in the absence it was negative. In particular, Fig. 1 reveals two interesting regimes. The first one concerns the removal of the Raman pulse walk-off by slow light when $\delta t=\Delta t_{L}$ $-\Delta t_{S L}=0$, obtained for the critical pump power $P_{c r}$ $=\beta_{2} \Omega_{R} \Delta \Omega_{R} A_{\text {effog }} g_{R}^{-1}$ [8]. In such conditions, Eq. (3) predicts that the frequency shift is unexpectedly nonzero and equal to [by setting $\delta_{S L}=\delta_{w}$ in Eq. (3)] 


$$
\delta \nu\left(\delta_{w}\right)=-\frac{\gamma P L_{w}}{\pi T_{0}}\left[\exp \left(-\delta_{w}^{2}\right)-1\right],
$$

yielding an up-frequency shift, which is of the order of $0.9 \mathrm{GHz}$ for the analytical value. Surprisingly, it is the exact opposite value of the down-frequency shift predicted by Eq. (2), i.e., in the absence of slow light. (See solid thin curve in Fig. 1.) This is counterintuitive: one may reasonably expect zero frequency shift, since there is no longer any walk-off between signal and pump pulses. Slow light exactly compensates for the GVD, leading to group-velocity matching between the two pulses. However, as this group velocity matching is not due to the absence of dispersion but to slow light, this nonzero frequency shift can be interpreted as resulting from the major difference between the phase and the group velocity variations in a slow-light medium. In such a case, we have to abandon the classical picture of the XPM theory where the chirp function exactly matches the pump intensity distribution. This is due to the fact that the pulse envelope is slowing down, whereas the chirp function is not affected by slow light, because it relies on the phase variation. Correspondingly, the signal phase propagates faster than the signal envelope and therefore travels across it. This results in a signal phase that is predominantly influenced by the pump trailing edge, leading to an up-frequency shift. This interpretation is verified by the numerical simulation. As shown in Fig. 2(b), even if the pulse walk-off is fully canceled by slow light, the signal still undergoes an up-frequency shift as shown in the inset of Fig. 2(b), as if the signal pulse predominantly interacted with the pump trailing edge. It can also be viewed in Fig. 2(b) that the zero point of the chirp function is still obtained for $\tau=\delta_{w} / 2$ as for Fig. 2(a), which clearly means that the chirp function is not affected by slow light.

The second case is even more intriguing and corresponds to the removal of the XPM-induced frequency shift at the pulse peak, as shown by the solid curves in Fig. 1. Equation (3) indeed predicts that the frequency shift can be set to zero when $L_{S L}=2 L_{w}$, i.e., $v_{g s}^{S L}=\left(v_{g p}+v_{g s}\right) / 2$, which corresponds to an input pump power that is half of the critical pump power $P=P_{c r} / 2$ as shown in Fig. 1. Note that this also implies that the chirp is less important than for both the previous cases at $P=P_{c r}$. As can be seen in Fig. 2 (c), the signal peak is now at the same temporal position as the zero point of the chirp function, whereas the pulse walk-off is no longer canceled ( $\delta t=30 \mathrm{ps}$ ). The inset of Fig. 2(c) shows that the signal spectrum no longer suffers from any frequency shift, despite the fact that the signal pulse interacts with the leading edge of the pump pulse. Note that factor 2 between the discussed regimes, i.e., zero walk-off and zero frequency chirp, is simply related to the fact that the zero point of the chirp function is set to $\tau=\delta_{w} / 2$, as predicted by Eq. (1). For pump power larger than $P_{c r}$, the pulse walk-off becomes negative due to the slowing down of the signal pulse, leading to an in- duced frequency shift that is predominantly positive as if it propagates in the anomalous dispersion regime.

In addition, the small discrepancies between analytical and numerical results in Fig. 1 rely mainly on the fact that the analytical expression of the Raman slow light delay is valid only in the steady-state regime, i.e., it assumes that the pump is a cw field and that the signal bandwidth is smaller than the Raman gain bandwidth [9]. As the signal center frequency can shift out of the gain bandwidth due to XPM, this leads to the decrease of the slowing-down of the signal pulse [see the insets of Figs. 2(a) and 2(b)]. It explains the fact that the numerical critical pump power needed to cancel the walk-off is higher than the analytical value. This is also consistent with the fact that the numerically calculated XPM-induced frequency shift is slightly greater than the analytical values.

In conclusion, we have theoretically demonstrated that in an optical fiber system with a narrowband Raman gain, not only the pulse walk-off but also the pulse frequency chirp can be all-optically tuned Negative or positive frequency shift can be achieved in this way simply by tuning the pulse walk-off using slow light. We have shown that this new implication is a direct consequence of the fact that the phase velocity is not affected by slow light compared to the group velocity. Note that we expect that this phenomenon cannot be observed in silica fibers nor with SBS, because of its backward nature, nor with SRS due to its broad gain bandwidth that leads to femtosecond optical delays much lower than the GVD delays [5].

The authors thank M. Gonzàlez-Herràez and L. Thévenaz for helpful discussions, and S. Coen for careful reading of the manuscript. This work is partially funded by the European INTERREG IV-A programme and COST action 299, and by the Conseil Régional de Franche-Comté.

\section{References}

1. R. W. Boyd and D. J. Gauthier, in Progress in Optics, E. Wolf, ed. (Elsevier, 2002), Vol. 43, Chap. 6, p. 495.

2. L. Thévenaz, Nat. Photonics, 2, 474 (2008).

3. Y. Okawachi, M. S. Bigelow, J. E. Sharping, Z. Zhu, A. Schweinsberg, D. J. Gauthier, R. W. Boyd, and A. L. Gaeta, Phys. Rev. Lett. 94, 153902 (2005).

4. K. Y. Song, M. G. Herráez, and L. Thévenaz, Opt. Express 13, 82 (2005).

5. J. E. Sharping, Y. Okawachi, and A. L. Gaeta, Opt. Express 13, 6092 (2005).

6. D. Dahan and G. Eisenstein, Opt. Express 13, 6234 (2005).

7. S. Lebrun, P. Delaye, R. Frey, and G. Rosen, Opt. Lett. 32, 337 (2003).

8. G. Fanjoux and T. Sylvestre, Opt. Lett. 33, 2506 (2008).

9. G. Fanjoux, J. Michaud, H. Maillotte, and T. Sylvestre, Phys. Rev. Lett. 100, 013908 (2008).

10. P. L. Baldeck, R. R. Alfano, and G. P. Agrawal, Appl. Phys. Lett. 52, 1939 (1988).

11. G. P. Agrawal, in Nonlinear Fiber Optics, 4th ed. (Academic, 2007). 